\title{
Flank Milling with Flat End Milling Cutters
}

\author{
Sanjeev Bedi \\ University of Waterloo, Department of Mechanical Engineering, Waterloo, \\ Ontario, Canada. N2L 3G1. (519) 888-4567, Fax (519) 888-6197 \\ Stephen Mann \\ University of Waterloo, Department of Computer Science, Waterloo, Ontario, \\ Canada. N2L 3G1. (519) 888-4567, Fax (519) 885-1208
}

\begin{abstract}
In this paper, we give a detailed mathematical understanding of flank milling with flat end cutters, which we use to develop a method for milling with such a cutter. This method slides the cutter along two rails, providing two points of contact on the surface.
\end{abstract}

Key words: 5-axis machining, flank milling

\section{Introduction}

Five-axis machining is gaining in popularity as it provides additional flexibility, which can be used to improve production efficiency and surface finish. Most work so far has focused on end-milling of doubly curved surfaces. Curvature matching methods match the curvature of the design surface to that of the tool surface and achieve reduction in production time of up to $85 \%$. Alternative techniques such as the multi-point method (MPM) use the dexterity of the 5 -axis machines to machine more than one point on the design surface at the same time $[1,2]$. The MPM results in slightly better production efficiencies than curvature matching methods but are harder to implement and less robust [3]. While much attention has been focused on machining with the bottom end of cutters, little research has been done on flank milling $[4,5]$, which can provide large productivity gains for such class of surfaces as are found in blades, fans, turbines and other engineering objects.

1 Email: sbedi@surya.uwaterloo.ca

2 Email: smann@cgl.uwaterloo.ca 
Flank milling of developable surfaces has been known and practiced for long. Flank milling of ruled surfaces in which curvature variation is minimum has also been published. This paper attempts to offer a detailed mathematical understanding of the flank milling with cylindrical flat end milling cutters. In particular, we show that the grazing curve of a cylinder is approximately a helix and thus it is not possible to exactly mill a ruled surface. We then show how to move a cylindrical tool tangentially along two curves (rails).

\section{Mathematics of Flank Milling}

An NC tool essentially is a cutting surface rotating at high velocity. This spinning cutter is moved along a work piece, removing any material in its path. The spindle rotation speed is much larger than the translation speed. Thus, for the type of tool we are considering, the volume traveled by a moving cylinder can approximate the material removed by a moving tool. Let the bottom center of a cylinder move along a curve $P(v)$, where $P(v)$ is a parametric curve. The cylinder is a rigid body so its axis does not change length. The movement of the tool axis, $a(v)$, is described using a unit sphere (spherical coordinates). Two points on the sphere are used to represent the orientation of the tool axis at the start and end of the motion. An interpolation of the sphere angle between these points specifies the trajectory of the tool axis. The interpolation may be linear or of higher degree. The resulting equation of the tool axis, which retains the length of the tool axis, is

$$
S(u, v)=P(v)+u a(\theta(v), \phi(v))
$$

where for simplicity we assume the axis is of unit length.

From this equation the direction of motion of any point $u$ on the tool axis can be calculated at any instance $v$ by differentiating $S(u, v)$ with respect to $v$ and substituting the proper $u$ and $v$ into the resulting equation.

At any given instance the bottom center and top center of the rigid cylindrical tool may move in directions that do not lie in the same plane. Let the velocity vectors for the top and bottom be $V_{t}$ and $V_{b}$ respectively. The velocity of in between points on the tool axis can be calculated by linearly interpolating $V_{t}$ and $V_{b}$ and is given by

$$
V=u V_{t}+(1-u) V_{b}=V_{t} u+V_{b}(l-u)
$$

where $l$ is the length of the cylindrical tool.

As the tool moves it cuts the material in front of it. Only a few points on the cylindrical surface of the tool are left un-machined as the tool moves away. The method to identify these points is discussed next. 


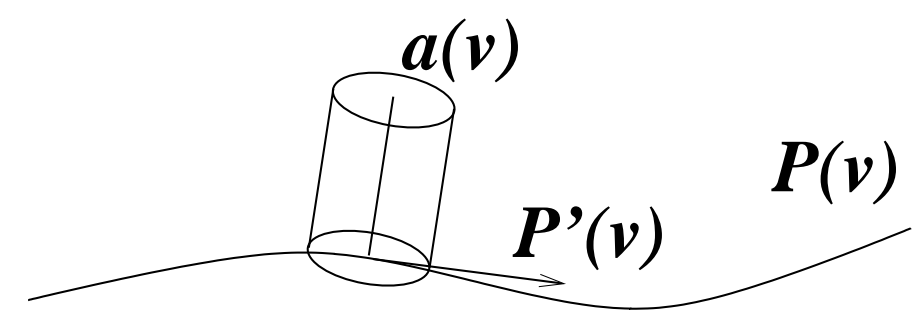

Fig. 1. Motion of tool along $P$.

\section{Grazing Points, Curves and Surface}

As a cylinder moves through stock the cylinder is in contact with numerous points on its surface. Most of these points lie towards the front of the tool and are machined away as the tool leaves its current position. Only those points for which the direction of motion is perpendicular to the normal of the cylindrical surface of the tool are left behind on the stock as a curve on the machined surface. These grazing points lie on the front side of the cutter relative to its motion. In earlier work, we showed how to compute points on the grazing curves on arbitrary surfaces of revolution $[6,7]$. Here, we will derive the equation for grazing curve on a cylinder, which turns out to be approximately a helix.

The velocity of all points on the tool axis is described in Equation 1. Each circular slice of the tool is a circular disk, where the velocity of the center of each disc is known. The velocity of a peripheral points on a disk is the sum of the translation of disk's center and a rotation about this center point. Thus $V_{s}=V+V_{r}$. The velocity component due to rotation, $V_{r}$, will lie in a plane tangent to the cylinder's surface. Thus, it is easy to identify those points on this disc at which the velocity of the surface point is perpendicular to surface normal. These are the points where the velocity of the tool axis or sphere center is perpendicular to the surface normal. Furthermore, these points lie at a distance $R$ (radius of cylinder) along the direction given by the cross product of $a(v)$ (the normal of the plane of the circle) and the velocity of the center of the disc, $V$. Typically this will result in two grazing points. By moving the disc from the bottom center to the top center numerous grazing points can be calculated and joined linearly to create the grazing curve at a given tool position. Different tool positions will result in other grazing curves, which can be linearly joined to give a grazing surface.

Thus the equation of the points that leave their impression on the stock is given by

$$
I(u, v)=S(u, v)+\frac{\left(\frac{d S(u, v)}{d v} \times a(v)\right)}{\left|\left(\frac{d S(u, v)}{d v} \times a(v)\right) R\right|} R=S(u, v)+R S_{n}(u, v)
$$

and we see that the surface machined by a single pass of a cylindrical tool is the offset of a ruled surface.

To understand the above equation consider a special case of a tool located at the origin in which the bottom center moves along the $x$-direction and the top center of the unit length 


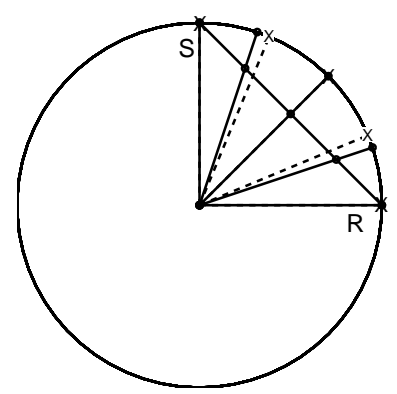

Fig. 2. Linear interpolation of $R$ and $S$ ('o')yields different points on the circle than linear interpolation of the angle ('x').

tool moves along the $y$-axis. The grazing point at the top lies on the $x$-axis at a unit distance along the $z$-axis whereas at the bottom center the grazing point lies along the $y$-axis in the $x y$-plane. The velocity of the in between points on the tool axis can be obtained by linear interpolation of the velocity of the top and bottom centers and the in between grazing points can be calculated to lie close to a helix on the cylindrical surface. Note that this curve is not exactly a helix, since as illustrated in Figure 2 linear interpolation of the velocity vectors is different from a linearly changing angle.

However, as we show in Section 5, this curve is nearly a helix that can be developed for the tool position corresponding to $v=v_{1}$ as follows. The bottom center of the tool is at $P\left(v_{1}\right)$ and the top center of the tool is at $P\left(v_{1}\right)+a\left(v_{1}\right)$. The corresponding velocities are $d P\left(v_{1}\right)$ and $d P\left(v_{1}\right)+d a\left(v_{1}\right)$. A reference frame can be established at $P\left(v_{1}\right)$ so that the $z$-axis is along the tool axis along $a\left(v_{1}\right)$. The $x$-axis is perpendicular to $z$-axis and to the velocity vector $d P\left(v_{1}\right)$. The $y$-axis can be determined from the $x$ - and $z$-axes. The helix can be described as

$$
[\operatorname{Rad} \cos (\theta), \quad \operatorname{Rad} \sin (\theta), \quad \theta C, \quad 1]
$$

in the local $x y z$-frame, where Rad is the radius of the cylinder. Its definition in the global frame can be obtained by multiplying it with a rotation matrix. The columns of the rotation matrix are the $x, y, z$ and the $P\left(v_{1}\right)$ vectors, which are generated as described above. It is possible to define a class of surfaces comprised of helical curves, but a moving cylindrical tool cannot machine all such surfaces. A moving cylindrical tool can only machine a small subset of such surfaces, for which the surface tangent is perpendicular to the radial arc of the helix.

The surface $S(u, v)$ of Equation 2 is a ruled surface, but its dependence on $\theta$ and $\phi$ gives it an awkward form. It is possible to represent $S(u, v)$ as a general ruled surface defined in terms of Bézier, B-spline, NURBS or algebraic terms. For a general ruled surface the length of the tool axis is not maintained as a constant and changes as $v$ varies. Thus, the velocity of any point along $u$ is a sum of its true velocity and the rate at which the tool length varies. The velocity of tool length lies along the tool axis and thus does not influence the calculation of the grazing points and curves, which will still lie on the offset surface to the ruled surface.

In general a cylindrical tool whose axis is sliding along the generating curves of a ruled 


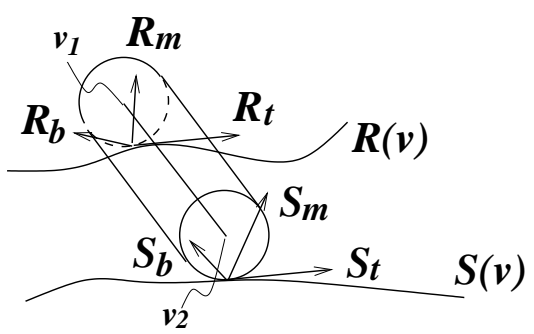

Fig. 3. Cylinder sliding along two rails.

surface will machine a surface offset by radius $R$ to the ruled surface. Thus if the surface swept by the axis of a cylindrical tool is known the surface machined by the tool can be determined. Furthermore, the above shows that the machined surface is composed of a series of nearly helical curves. This shows that we can not machine a ruled surface exactly using 5 -axis cylindrical flank milling cutting unless the ruled surface is developable.

\section{Cylindrical Tool Sliding on Two Rails}

One situation encountered in industry is that two curves on the machined surface are known. These curves could be the top and bottom profiles of a blade for example. The shape of the surface is not known and is to be determined by a tool sliding along the generating rails. Let the rails be defined by two parametric curves as $R(v)$ and $S(v)$. The parameter $v$ relates the contact points on $R(v)$ to those on $S(v)$.

Rubio et al. described two methods for placing the tool on points $R(v)$ and $S(v)$, but not tangent to both points [4]. Later, Redonnet et al. devised a method for placing the tool tangent to both curves [5], but the points of tangency would be for different values of $v$ (e.g., at placing the tool tangent to $R\left(v_{0}\right)$ and $\left.S\left(v_{1}\right)\right)$. In this work, we will give a method for placing the tool tangent to the two curves at the same parameter value.

Let $R_{t}(v), R_{m}(v)$ and $R_{b}(v)$ describe the tangent, main normal and binormal of the Frenet frame at $R(v)$. Similarly, let $S_{t}(v), S_{m}(v)$ and $S_{b}(v)$ describe the tangent, main normal and binormal of the Frenet frame at $S(v)$. When the tool is positioned to be tangential at $R(v)$ and $S(v)$ the tool axis of the cylindrical tool will pass through the plane described by $R_{m}(v)$ and $R_{b}(v)$ near $R(v)$ as illustrated in Figure 3. It will also pass through the plane described by $S_{m}(v)$ and $S_{b}(v)$ near $S(v)$. Let the intersection point in plane $R_{m}(v)$ and $R_{b}(v)$ be $v_{1}$ and in plane $S_{m}(v)$ and $S_{b}(v)$ be $v_{2}$. Both $v_{1}$ and $v_{2}$ are located at a distance equal to the tool radius $R a d$ from $R(v)$ and $S(v)$, respectively. The orientation of the tool axis is given by $v_{1}-v_{2}$. Note that since $R$ and $S$ are space curves, the vector from $R(v)$ to $v_{1}$ is not parallel to any of the Frenet directions; however it does lies in the plane perpendicular to $R_{t}(v)$ and can be described as

$$
v_{1}-R(v)=R a d \cos (\theta) R_{m}(v)+\operatorname{Rad} \sin (\theta) R_{b}(v)
$$


Similarly,

$$
v_{2}-S(v)=\operatorname{Rad} \cos (\beta) S_{m}(v)+\operatorname{Rad} \sin (\beta) S_{b}(v) .
$$

The vector $\left(v_{1}-R\right)$ must be perpendicular to the axis $\left(v_{1}-v_{2}\right)$ and similarly, the vector $\left(v_{1}-S\right)$ must also be perpendicular to the axis $\left(v_{1}-v_{2}\right)$. This gives the following equations.

$$
\begin{aligned}
& \left(v_{1}-v_{2}\right) \cdot\left(\cos (\theta) R_{m}(v)+\sin (\theta) R_{b}(v)\right)=0 \\
& \left(v_{1}-v_{2}\right) \cdot\left(\cos (\beta) S_{m}(v)+\sin (\beta) S_{b}(v)\right)=0 .
\end{aligned}
$$

After expansion and simplification the equations become

$$
\begin{aligned}
\cos (\theta) p_{1}+\sin (\theta) q_{1}+\text { Rad } & =0 \\
\cos (\beta) p_{2}+\sin (\beta) q_{2}+\text { Rad } & =0 .
\end{aligned}
$$

where

$$
\begin{aligned}
p_{1} & =\left((R-S) \cdot R_{m}(v)-S_{b} \cdot R_{m}(v) \operatorname{Rad} \sin (\beta)-S_{m} \cdot R_{m}(v) \operatorname{Rad} \cos (\beta)\right) \\
q_{1} & =\left((R-S) \cdot R_{b}(v)-S_{b} \cdot R_{b}(v) \operatorname{Rad} \sin (\beta)-S_{m} \cdot R_{b}(v) \operatorname{Rad} \cos (\beta)\right) \\
p_{2} & =\left((R-S) \cdot S_{m}(v)-R_{b} \cdot S_{m}(v) \operatorname{Rad} \sin (\theta)-R_{m} \cdot S_{m}(v) \operatorname{Rad} \cos (\theta)\right) \\
q_{2} & =\left((R-S) \cdot S_{b}(v)-R_{b} \cdot S_{b}(v) \operatorname{Rad} \sin (\theta)-R_{m} \cdot S_{b}(v) \operatorname{Rad} \cos (\theta)\right)
\end{aligned}
$$

To find $\theta$ and $\beta$, the above equations (3) are solved with the following equations simultaneously:

$$
\begin{aligned}
\sin ^{2}(\theta)+\cos ^{2}(\theta) & =1, \\
\sin ^{2}(\beta)+\cos ^{2}(\beta) & =1 .
\end{aligned}
$$

This gives a system of four equations in four unknowns, which we solve using numerical methods. These equations will in general have four solutions (one for each possibility of each end of the cylinder being above or below the corresponding rail).

\section{Example}

The above method was implemented in Maple, a symbolic algebra package [8], and the above four equations were solved simultaneously for a specific case in which $R(v)$ and $S(v)$ are two 

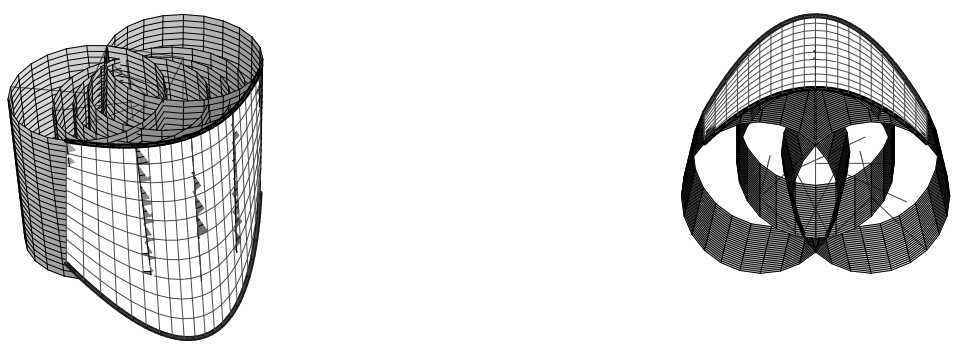

Fig. 4. Cylinder moving along two rails.

quadratic Bézier curves given by the following control points:

$$
R_{0}=[1,0,1] \quad R_{1}=[0,0,1] \quad R_{2}=[0,1,1],
$$

while the second curve had control points

$$
S_{0}=[1,0,0] \quad S_{1}=[-.25,-.25,-1] \quad S_{2}=[0,1,0] .
$$

The trajectory of a cylindrical tool of radius 0.5 , moving on the rails $R(v)$ and $S(v)$ is a ruled surface. The surface created by the moving tool can be determined by the method described earlier (Figure 4). The rails on which the tool slides are the boundaries of this surface and are also shown in the figure as thick solid lines. Note that since the distance between the rails varies, only one end of the cylinder can touch a rail - the other rail must touch else where on the cylinder, as seen in the left side of Figure 4.

If this method is used to machine a ruled surface by rolling the cylindrical tool along the generating curves of the surface, then the machined surface will match the ruled surface only along the generating curves. The maximum deviation will occur along the mid-curve of the ruled surface where the helical segment deviates most from the chord joining the same start and end points. Let $P_{a}$ and $P_{b}$ be the two points on the tool axis and let $S_{a}$ and $S_{b}$ be the corresponding points on the machined surface. Then the angle between them is given by

$$
\Delta:=\arccos \left(\left(S_{a}-P_{a}\right) \cdot\left(S_{b}-P_{b}\right) / \operatorname{Rad}^{2}\right) .
$$

Then the deviation is given by

$$
\epsilon:=\operatorname{Rad}^{2} \cos (\Delta / 2) .
$$

The deviation between the ruled surface and the swept surface is plotted in Figure 5, with a maximum distance of 0.0027 . If we reduce the tool radius to 0.25 , the maximum distance reduces to 0.0013 (the plot looks similar). Further, the distance is similar whether we use 


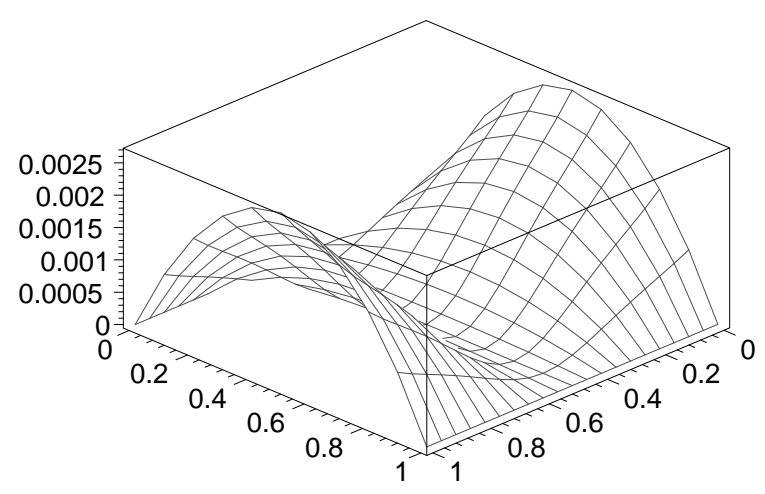

Fig. 5. Error in milling with flank mill.

the actual grazing curve or the helical approximation (the maximum angle between the top and bottom velocity vectors of the tool axis is .2 radians or 11 degrees, and the error between helix and actual grazing curve is .000033). Thus, the helix may provide an acceptable approximation to the true grazing curve. Further note that the step size in this example is large; using step sizes more common in machining may result in a machined surface that is an acceptable approximation to a ruled surface.

It is possible to generate the tool path relative to the mid curve of the ruled surface by offsetting the tool along the normal at the mid curve and aligning the tool axis along the ruled line. With this method the machined surface will match the ruled surface exactly along the mid curve and the maximal error will be at the generating curves. Alternatively, the offset could be altered by half the measure of the calculated error $\epsilon$, thereby reducing the maximal error between the machined surface and the ruled surface.

\section{Discussion}

In this paper, we have give the equation for the surface milled by a cylindrical cutter when flank milling. Although the equation itself is fairly complex, we can find the grazing curve on the surface at any position in its path. This curve is close to but not exactly a helix. Except in special cases, the grazing curve is not a line, showing that a cylindrical tool can not mill a ruled surface exactly. However, for small tool steps the machined surface may be an acceptable approximation to a ruled surface. Further, the observation that the grazing curve is almost a helix should be useful for CAD systems that have helixes but not general algebraic curves.

Using the method for finding the grazing curve, we have shown how to implement a method for moving a cylindrical cutter along two rails. To test this method, we used Maple to solve the equations numerically. It may be possible, however, that there is a closed form solution to these equations. 


\section{References}

[1] A. Warkentin, F. Ismail, and S. Bedi. Intersection approach to multi-point machining of sculptured surfaces. Computer Aided Geometric Design, 15(6):567-584, 1998.

[2] A. Warkentin, F. Ismail, and S. Bedi. Comparisons between multi-point and other 5-axis tool positioning strategies. International Journal of Machine Tools and Manufacture, 40:185-208, 2000.

[3] A. Warkentin, F. Ismail, and S. Bedi. Multi-point tool positioning strategy for 5-axis mashining of sculptured surfaces. Computer Aided Geometric Design, 17(1):83-100, 2000.

[4] W. Rubio, P. Lagarrigue, G. Dessein, and F. Pastor. Calculation of tool paths for a torus mill on free-form surfaces five-axis machines with detection and elimination of interference. The International Journal of Advanced Manufacturing Technology, 14:13-20, 1998.

[5] J.-M. Redonnet, W. Rubio, and G. Dessein. Side milling of ruled surfaces: Optimum positioning of the milling cutter and calculation of interference. International Journal for Advanced Manufacturing Technology, 14:459-465, 1998.

[6] D. Roth, S. Bedi, F. Ismail, and S. Mann. Surface swept by a toroidal cutter during 5-axis machining. Computer Aided Design, 33(1):57-63, 2001.

[7] S. Mann and S. Bedi. Generalization of the imprint method to general surfaces of revolution for NC machining. Computer-Aided Design, 2001. accepted for publication.

[8] M.B. Monagan, K.O. Geddes, G. Labahn, and S. Vorketter. The Maple Programming Guide. Springer-Verlag, 1996. 